Full length article

\title{
Effect of imperfect CSI on interference alignment in multiple-High Altitude Platforms based communication
}

\author{
Sudheesh P.G. ${ }^{a, *}$, Navuday Sharma $^{\text {b }}$, Maurizio Magarini ${ }^{\text {b }}$, P. Muthuchidambaranathan ${ }^{a}$ \\ a Department of Electronics and Communication Engineering, National Institute of Technology, 620015 Tiruchirappalli, India \\ b Dipartimento di Elettronica, Informazione e Bioingegneria, Politecnico di Milano, 20133 Milano, Italy
}

\section{A R T I C L E I N F O}

\section{Article history:}

Received 12 August 2017

Received in revised form 7 November 2017

Accepted 8 November 2017

Available online $\mathrm{xxxx}$

\begin{abstract}
A B S T R A C T
Interference Alignment (IA) offers maximum sum-rate in a wireless X channel. Though IA was proposed for maximizing sum-rate, its application for exploiting high data rate in air-to-ground communication has not been explored much. In this paper, the application of IA in a High Altitude Platform (HAP) to Ground Station (GS) communication is considered. Recent studies suggest that IA provides maximum sum-rate for a $2 \times 2$ transmitter-receiver system. However, independent channels are required to achieve IA conditions. The application of IA is proposed here for a generalized channel in an HAP-to-GS communication link that takes into account angle-of-departure and angle-of-arrival at the transmitter and at the receiver, respectively. We verify the minimum distance criteria in receiving nodes to achieve independent channels. Receivers are placed at optimal distance for best error performance. Furthermore, in view of an actual scenario, we investigate the effect of imperfect CSI, resulting from changes in imperfection in HAP's stabilization, in the performance of our model. The performance, in terms of Bit Error Rate (BER), is presented for IA and non-IA based communication. For this purpose, an analytical expression is developed for the probability of error. A perfect match is shown between the error rate measured with Monte Carlo simulations and the error probability found using the derived analytical expressions.
\end{abstract}

(C) 2017 Elsevier B.V. All rights reserved.

\section{Introduction}

Among multiple transmitter-receiver communication systems, the interference channel and the $\mathrm{X}$ network with $K$ users have found considerable attention [1]. In contrast to the interference channel, where $K$ transmitter-receiver pairs are considered, in an $\mathrm{X}$ network each transmitter has data intended for each receiver. An $X$ network behaves as a generic model by addressing other channel models such as interference channel, $\mathrm{Z}$ channel and $\mathrm{Y}$ channel as its special case [2].

When $K=2$, the $\mathrm{X}$ network is referred to as $\mathrm{X}$ channel. As shown in [3] the capacity of an X channel at high Signal-to-Noise Ratio (SNR) can be approximated as

$C(\mathrm{SNR})=d \cdot \log (\mathrm{SNR})+\mathcal{O}(\log (\mathrm{SNR}))$,

where $d$ corresponds to the number of Degrees of Freedom (DoF), which is also referred to as the multiplexing gain or capacity pre$\log$ factor. The maximum DoF was found to be $4 / 3$ for an $X$ channel [4] and, although many schemes are applicable in X channel to

\footnotetext{
* Corresponding author.

E-mail address: 408114001@nitt.edu (Sudheesh P.G.).
}

achieve it, IA attains such an upper bound using linear precoding at the transmitter [5].

This means that if we consider an X channel defined by a multiple Base Station (BS) communication system, IA claims to achieve higher sum-rate. In [2], the authors propose sharing a huge amount of data, e.g., a multimedia file, by two BSs to communicate with a mobile user that is under the vicinity of both of them. Hence, $X$ channel finds interesting applications and IA allows us to achieve the sum-rate upper bound on it. In this scenario, we consider the application of IA for the transmission from the High Altitude Platform (HAP) to the Ground Station (GS).

The main advantage of using HAPs is the possibility of deploying directional antennas, which leads to efficient bandwidth reuse [6]. Thus, HAPs offer better spectral efficiency over satellite communication. The location of HAPs in altitude is between 17 and 22 $\mathrm{km}$, which leads to a Line-of-Sight (LoS) channel in the HAP-to-GS link $[7,8]$. The two frequency bands allotted for communication are $31 \mathrm{GHz}$ and $48 \mathrm{GHz}$ [9]. In this work, we explore the possibility of applying IA for HAP-to-GS communication. The system consists of two HAPs serving two GSs with overlapping service areas. Though the maximization of the capacity in a single HAP-to-GS link has recently gained considerable attention, the same problem has not been addressed by previous studies for the case of multiple HAPs. 
Since the above mentioned system forms an X channel one may think of using IA to maximize the capacity.

A crucial role in the $\mathrm{X}$ channel is played by multiple-input multiple-output (MIMO) transmission, which dictates the nature of the channel. It is indeed a requirement of the communication system to have a full rank MIMO channel matrix associated with each link to exploit the property of eigenmode transmission. This is a very important property to achieve high capacity in MIMO communication. Hence, in addition to IA, having independent MIMO channels in HAP-to-GS communication results in higher sum-rate for the system. However, as HAPs are located at a higher position, having independent channels is a bottleneck for the design of the communication system. In [10], Madhow et al. suggest separating the receiver nodes to provide independent channels. This separation is a function of center frequency, distance between the transmitter and receiver, and the antenna separation at transmitter and receiver [10-12]. The channel model proposed in [10] is valid for large distance LoS wireless communication. Hence, to achieve zero correlation between MIMO channels associated with different links the receivers must be spaced apart an adequate distance. Since it is impossible to achieve IA in single rank MIMO communication, we suggest using a more generalized channel model for HAP-to-GS communication that includes the Angle-of-Arrival at the receiver $\left(\mathrm{AoA}_{R}\right)$ and the Angle-of-Departure at the transmitter $\left(\mathrm{AoD}_{T}\right)$.

An important assumption made in [13] is that Channel State Information (CSI) is globally available at each transmitting and receiving node. Knowledge of global CSI enables us to write closed form expressions for the pre-coding and Zero-Forcing (ZF) matrices. This means that, the performance of IA is heavily dependent on precise CSI. In our recent work [14], we have considered the minimum receiver separation required to achieve maximum multiplexing gain provided by IA in multiple HAP based system. In addition, we have proposed an LoS channel model to monitor the performance of the system. An important assumption in [14] is that perfect CSI is available for every transmitter and receiver. However, precise CSI may not be available at the transmitter due to change in location of HAPs or imperfections in station keeping. To this end, in the present work, we investigate the effect of imperfect CSI, which may occur due to the imperfections in station keeping of HAPs.

The paper is structured as follows. The system model and the considered channel are reviewed in Section 2. In Section 3 we give a detailed explanation of IA for X channel by considering HAP-toGS links each defined by a MIMO channel. In Section 4 we present numerical results and, finally, we draw conclusions in Section 5.

\section{Interference alignment for the existing long distance LoS channel}

In this section we discuss the existing long distance LoS channel model proposed in [10] and investigate the possibility of applying IA in the resulting $X$ channel. Multiple antennas are used at both HAP and GS ends. We also find the minimum separation required between the receivers to achieve independent channels, that is an important factor in capacity maximization.

\subsection{Background}

In case of terrestrial communication, the channel is modeled as Rayleigh in urban areas and Rician in suburban areas. This is not the same for HAP-to-GS channel [15]. For urban conditions, HAP-to-GS channel experiences Rician fading due to the presence of LoS path. In suburban areas, a Rayleigh fading is experienced due to the presence of reflected signals that are stronger than LoS [15]. A generalized approach will be to use a Rician distribution where both LoS and Non-LoS (NLOS) paths are considered. Let $N_{R}$ and $N_{T}$ denote the number of receiving and transmitting antennas, respectively. The resulting $N_{T} \times N_{R}$ MIMO channel model is

$\mathbf{H}=\sqrt{\frac{\kappa}{(1+\kappa)}} \mathbf{H}_{L o S}+\sqrt{\frac{1}{(1+\kappa)}} \mathbf{H}_{N L O S}$,

where $\mathbf{H}_{\text {LoS }}$ represents shadowed free space propagation loss and $\mathbf{H}_{N L O S}$ represents only the NLoS path. By denoting as $\sigma_{L O S}^{2}$ and $\sigma_{N L O S}^{2}$ the power associated with LoS path and NLoS path, respectively, the Rician factor $\kappa$ is given by [16]

$\kappa=\frac{\sigma_{\text {LoS }}^{2}}{\sigma_{N L O S}^{2}}$.

Neglecting the distance between transmitter and receiver, the LoS channel is given by [16]

$\mathbf{H}=\left[\begin{array}{c}1 \\ e^{j 2 \pi \frac{d_{R}}{\lambda} \sin \left(\mathrm{AoA}_{R}\right)} \\ \vdots \\ e^{j 2 \pi \frac{d_{R}}{\lambda}(M-1) \sin \left(\mathrm{AoA}_{R}\right)}\end{array}\right]\left[\begin{array}{c}1 \\ e^{j 2 \pi \frac{d_{T}}{\lambda} \sin \left(\mathrm{AoD}_{T}\right)} \\ \vdots \\ e^{j 2 \pi \frac{d_{T}}{\lambda}(N-1) \sin \left(\mathrm{AoD}_{T}\right)}\end{array}\right]^{T}$,

where $\lambda$ is the wavelength, $d_{R}$ and $d_{T}$ represent the antenna spacing in the receiver and transmitter, respectively. The two angles $\mathrm{AoA}_{R}$ and $\mathrm{AoD}_{T}$ provide crucial information on the position of HAP with respect to the GS, as shown in Fig. 1. Mostly, HAPs possess an elevation angle of $60-80^{\circ}$. Then, in that case, $\mathrm{AoA}_{R}$ and $\mathrm{AoD}_{T}$ can be taken as $10-30^{\circ}$. Since $\mathbf{H}_{N L o S}$ represents only the NLoS path, the resulting NLoS MIMO channel follows a Rayleigh distribution. Therefore, $A_{0} A_{R}$ and $A_{0 D}$ has direct impact on the LoS nature of the channel.

The model of a Three-Dimensional (3D) MIMO channel is considered in [17] for a planar array of antennas for a single HAP that communicate with several randomly placed GSs. Similar work in [11] considers a channel model that is suitable for long distance wireless communication. The effective long distance channel from transmitter $n$ to receiver $m$ is modeled as

$h_{\text {LoS }}(m, n)=\frac{\lambda}{4 \pi R} \exp \left(-j \frac{2 \pi}{\lambda}\left(R+\frac{\left(m d_{T}-n d_{R}\right)^{2}}{2 R}\right)\right)$,

where $R$ denotes the transmitter-receiver distance. However, the directional beam from a transmitter is obtained using sub arrays with beamsteering. Since the spacing between antennas in the transmitter and receiver is less, the above said arrangement provides array gain rather than spatial multiplexing gain.

\subsection{Imperfect CSI}

With a transmitter-receiver separation of $17-22 \mathrm{~km}$, and absence of precise station keeping, it is difficult to obtain an accurate CSI, which is necessary to perform IA. In addition, ZF and precoding matrices are designed from locally estimated CSI. Hence, it is obvious to assume imperfection in the obtained CSI. Following [18], in this paper we relate the imperfect CSI $(\hat{\mathbf{H}})$ to the actual CSI $(\mathbf{H})$ using a correlation coefficient $\rho$ as

$\hat{\mathbf{H}}=\rho \mathbf{H}+\bar{\rho} \Phi$,

where $\rho \in(0,1), \bar{\rho}=\sqrt{1-\rho^{2}}$, and $\Phi$ is normally distributed with $\mathcal{N}(0,1)$. To analyze the effect of imperfect CSI on our model, precoding and ZF matrices are designed from the locally available $\hat{\mathbf{H}}$. 


\subsection{Long distance LoS channel and receiver separation}

Here, we investigate the minimum receiver node separation that is required to avoid channel correlation. We look at transmitted power and attenuation introduced by the HAP-to-GS link influencing the transmitter design. Concerning with the constraint on the transmitted power, this is overcome in unmanned aircrafts as demonstrated in Helios [19], where solar cells with a wing span of $35-70 \mathrm{~m}$ were employed. The mission was designed to last for about 6 months and, therefore, no extra human interference is needed throughout this period of time. Hence, in our paper, we do not consider any power constraint.

We begin with LoS MIMO as a basic system to model the communication for the HAP-to-GS link. For an HAP communication, maximum path loss happens due to free space propagation. Though [10] uses multiple antennas both at the transmitter and the receiver, the goal is to attain the array gain in place of the spatial multiplexing gain. It is then required to have higher capacity in the system to meet the present and future demands. To this end, [11] and [12] suggest that spatial multiplexing can still be used for the long distance LoS channel, provided that the separation between antennas in the sub-arrays is large enough to provide zero correlation between subsequent channels. However, inter-antenna separation distance varies with center frequency and separation between transmitter and receiver. In [11], authors give a numerical approach to calculate inter-antenna spacing to achieve full rank channel matrix in LoS MIMO channel. The calculation is done with the goal of nullifying the correlation between columns of LoS MIMO channel. By exploiting the same logic, it is possible to find inter node distance at receiver to obtain independent channel. From [11], we have

$d_{T} d_{R}=n \frac{R \lambda}{D o F}$,

where $n$ can be any integer except the DoF.

Here, we find the required inter-antenna spacing for HAP-to-GS communication. We consider the operating band as $48 \mathrm{GHz}$ and height at which HAP is located as $18 \mathrm{~km}$. Hence, from (7), with node separation at transmitter side $d_{T}=1 \mathrm{~m}$ and $D o F=2$, we find the inter node separation at the receiver as $d_{R}^{\text {opt }}=56.2 \mathrm{~m}$. This means that it is possible to exploit spatial multiplexing in HAP communication if the receiver antenna separation is $56.2 \mathrm{~m}$, which further means maximum capacity transmission.

\section{Role of IA for HAP based transmission}

We consider two HAPs serving a common area where there are two receivers. This essentially forms a $2 \times 2 \mathrm{X}$ channel. Hence, we investigate the performance by considering the MIMO X channel depicted in Fig. 1.

\subsection{Background of interference alignment}

We consider the situation where each transmitting and receiving node is equipped with three antennas. The model for the signal vector received by the first and second GS is

$\mathbf{y}_{1}=\mathbf{H}_{11} \mathbf{x}_{1}+\mathbf{H}_{12} \mathbf{x}_{2}+\mathbf{n}_{1}$,

and

$\mathbf{y}_{2}=\mathbf{H}_{21} \mathbf{x}_{1}+\mathbf{H}_{22} \mathbf{x}_{2}+\mathbf{n}_{2}$,

respectively. In the above equations, $\mathbf{x}_{i}$ denotes the $3 \times 1$ vector transmitted by user $i, \mathbf{H}_{j i}$ is the $3 \times 3$ channel matrix between $i$ th transmitter and $j$ th receiver, with $i, j \in\{1,2\}$. The elements of $\mathbf{H}_{j i}$ are assumed to have a unit variance. The entries of the $3 \times 1$ noise

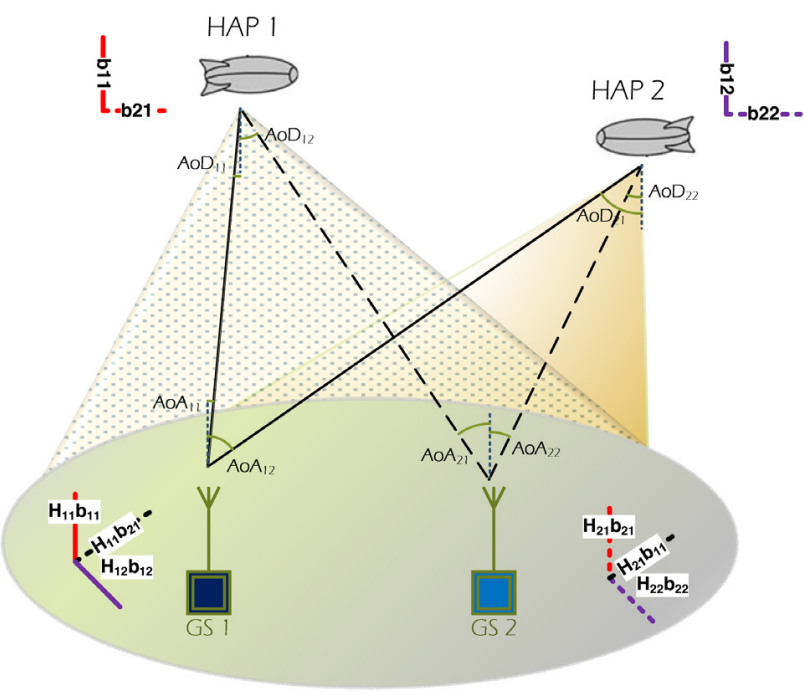

Fig. 1. HAP communication architecture with overlapped coverage area.

vector $\mathbf{n}_{i}$ are independent and normally distributed as $\mathcal{N}\left(0, \sigma_{n}^{2}\right)$. The two transmitted vectors are given by

$\mathbf{x}_{1}=\mathbf{b}_{11} x_{11}+\mathbf{b}_{21} x_{21}$

and

$\mathbf{x}_{2}=\mathbf{b}_{12} x_{12}+\mathbf{b}_{22} x_{22}$

where $x_{j i}$ is the message from transmitter $i$ to receiver $j$ that is shaped by the beamforming vector $\mathbf{b}_{j i}$. By replacing (10) and (11) in (8) and (9), respectively, we have

$$
\begin{aligned}
\mathbf{y}_{1}= & \mathbf{H}_{11} \mathbf{b}_{11} x_{11}+\mathbf{H}_{11} \mathbf{b}_{21} x_{21} \\
& +\mathbf{H}_{12} \mathbf{b}_{12} x_{12}+\mathbf{H}_{12} \mathbf{b}_{22} x_{22}+\mathbf{n}_{1} \\
\mathbf{y}_{2}= & \mathbf{H}_{21} \mathbf{b}_{11} x_{11}+\mathbf{H}_{21} \mathbf{b}_{21} x_{21} \\
& +\mathbf{H}_{22} \mathbf{b}_{12} x_{12}+\mathbf{H}_{22} \mathbf{b}_{22} x_{22}+\mathbf{n}_{2} .
\end{aligned}
$$

The IA condition is satisfied when the interfering signals span the same subspace [1] according to

$\operatorname{SPAN}\left\{\mathbf{H}_{12} \mathbf{b}_{22}\right\}=\operatorname{SPAN}\left\{\mathbf{H}_{11} \mathbf{b}_{21}\right\}$

and

$\operatorname{SPAN}\left\{\mathbf{H}_{22} \mathbf{b}_{12}\right\}=\operatorname{SPAN}\left\{\mathbf{H}_{21} \mathbf{b}_{11}\right\}$.

Beamforming vectors are chosen as

$\mathbf{b}_{22}=\mathbf{H}_{12}^{-1} \mathbf{H}_{11} \mathbf{b}_{21}$

and

$\mathbf{b}_{12}=\mathbf{H}_{22}^{-1} \mathbf{H}_{21} \mathbf{b}_{11}$.

Using (16) and (17), beamforming vectors are formed in the transmitter and, as a result, interference vectors are aligned in the receivers. A zero-forcing receiver is used to separate undesired signals from the desired ones.

\subsection{Achieving IA in rank 1 MIMO channel}

It is required to choose $\mathbf{b}_{21}$ and $\mathbf{b}_{11}$ as two independent vectors and, therefore, $\mathbf{b}_{22}$ and $\mathbf{b}_{12}$ are obtained from (16) and (17). For illustrating the communication along a rank 1 channel, we first consider the case of receiver 1 . With $\mathbf{b}_{11}$ defined, with rank 1 
channel matrix, $\mathbf{b}_{12}$ is nothing but an amplified/attenuated version of $\mathbf{b}_{11}$. This essentially means that (17) results in non independent vectors. As a result, $\mathbf{H}_{22} \mathbf{b}_{12}$ and $\mathbf{H}_{21} \mathbf{b}_{11}$ are aligned along the same direction in receiver 1 , which further means that independent vectors intended for zero-forcing data from two transmitters become dependent.

This is the same as receiver 2 . Choosing $\mathbf{b}_{22}$ as attenuated/amplified version of $\mathbf{b}_{21}$ leads to aligned vectors at receiver 2 . Actually, due to the presence of a rank 1 channel, the IA decoding is compromised. In this scenario, we propose a more generalized LoS channel in 3.3 for long distance wireless communication.

\subsection{Achieving IA through generalized channel model}

Channel modeling is carried out by considering an LoS path between HAP and GS. Hence, the effect of shadowing is less, compared to terrestrial communication. In addition, the path loss is also considered, as in [10,11].

We consider a MIMO link where transmitting and receiving antennas are separated by a distance of $\lambda / 2$. Now, it can be easily inferred that the channel is indeed dependent on AoA, AoD and $R$. Under these circumstances, the complex channel gain for LoS path, $h_{\text {LoS }}(m, n)$ is modeled as

$$
\begin{aligned}
h_{\text {LoS }}(m, n)= & \frac{\lambda}{4 \pi R} \exp \left(-j \frac{2 \pi}{\lambda}\left(m \frac{\lambda}{2} \sin (A o A)\right.\right. \\
& +n \frac{\lambda}{2} \sin (A o D) \\
& \left.\left.+R+\frac{\left(m \frac{\lambda}{2}-n \frac{\lambda}{2}\right)^{2}}{2 R}\right)\right),
\end{aligned}
$$

where $n=1, \ldots, N_{t}$ and $m=1, \ldots, N_{r}$. Hence, the channel coefficients differ due to the AoA and AoD. As a result the beamforming vectors to perform IA will be independent and this, in turn, forms independent vectors at the receiver. Here, data from both the transmitters are now aligned over independent vectors, and information can be recovered. Hence with this generalized channel model IA can be carried out.

\subsection{Error performance under imperfect CSI}

In this subsection, we derive the SNR and capacity for the system with imperfect CSI. We begin by generalizing the received signals given in (12) and (13) and by deriving the SNR, which is further used to get expression for capacity.

The received signal is given as

$\mathbf{y}_{i}=\sum_{j=1}^{K} \mathbf{H}_{i j} \sum_{l=1}^{K} \mathbf{b}_{l j} x_{l j}+\mathbf{n}_{i}, \quad i, j=1, \ldots, K$.

where $x_{i j}$ is the message to be transmitted from transmitter $j$ to receiver $i$ and $\mathbf{b}_{i j}$ is $x_{i j}$ 's associated beamforming vector.

The above equation can be rewritten as

$\mathbf{y}_{i}=\sum_{j=1}^{K} \mathbf{H}_{i j} \mathbf{b}_{i j} x_{i j}+\sum_{j=1}^{K} \sum_{l=1, l \neq i}^{K} \mathbf{H}_{i j} \mathbf{b}_{l j} x_{l j}+\mathbf{n}_{i}$,

where the first and the second term at the RHS are the signal vector intended for the $i$ th receiver and the interference affecting it, respectively. In the receiver, the $k$ th desired signal in $i$ th receiver is obtained by multiplying with a zero-forcing vector $\mathbf{w}_{i k}$. Therefore, the $k$ th desired signal component is given by

$\hat{\mathbf{x}}_{i k}=\mathbf{w}_{i k}^{H} \mathbf{y}_{i}$,

$\hat{\mathbf{x}}_{i k}=\underbrace{\mathbf{w}_{i k}^{H} \mathbf{H}_{i k} \mathbf{b}_{i k} x_{i k}}_{\text {Desired signal }}+\underbrace{\mathbf{w}_{i k}^{H} \sum_{\substack{j=1,1 \\ j \neq k}}^{K} \mathbf{H}_{i j} \mathbf{b}_{i j} x_{i j}}_{\begin{array}{c}\text { Interference from } \\ \text { other desired vectors }\end{array}}$

$$
+\underbrace{\mathbf{w}_{i k}^{H} \sum_{j=1}^{K} \sum_{l=1, l \neq i}^{K} \mathbf{H}_{i j} \mathbf{b}_{l j} x_{l j}}_{\begin{array}{c}
\text { Interference from } \\
\text { undesired vectors }
\end{array}}+\underbrace{\mathbf{w}_{i k}^{H} \mathbf{n}_{i}}_{\text {noise term }},
$$

where $K$ denotes the number of desired symbols in the receiver, which is 2 for a $2 \times 2$ system, as illustrated in Fig. 1 .

The SNR of the $k$ th stream is given by

$$
\gamma_{k}=\frac{\left|\mathbf{w}_{i k}^{H} \mathbf{H}_{i k} \mathbf{b}_{i k} x_{i k}\right|^{2}}{\left|\mathbf{w}_{i k}^{H} \sum_{j=1, j \neq k}^{K} \mathbf{H}_{i j} \mathbf{b}_{i j} x_{i j}\right|^{2}+\left|\mathbf{w}_{i k}^{H} \sum_{j=1}^{K} \sum_{l=1, l \neq i}^{K} \mathbf{H}_{i j} \mathbf{b}_{l j} x_{l j}\right|^{2}+\sigma^{2}} .
$$

Note that, the $\mathbf{H}_{i j} \in i, j$ follows (2) for ideal channel case, and follows (6) for imperfect channel case. However, $\rho=1$ denotes that channel is perfect and (6) can be equated to (2). Now, from Shannon's theorem, we know that the capacity of the system is given by

$\mathbf{C}=\sum_{k=1}^{K} \log \left(1+\gamma_{k}\right)$.

Therefore, by substituting Eqs. (6) and (22) in (23), the behavior of channel imperfection in system's capacity can be found.

Similarly, the conditional Symbol Error Rate (SER) can be derived from the SNR for $M-Q A M[20]$,

$$
\begin{aligned}
S E R_{\text {cond }}= & \frac{4}{\pi}\left(1-\frac{1}{\sqrt{M}}\right) Q\left(\sqrt{2 g_{Q A M} \gamma}\right) \\
& -\frac{4}{\pi}\left(1-\frac{1}{\sqrt{M}}\right)^{2} Q\left(\sqrt{2 g_{Q A M} \gamma}\right)^{2} \\
S E R_{\text {cond }}= & \frac{4}{\pi}\left(1-\frac{1}{\sqrt{M}}\right)\left[\frac{1}{\sqrt{M}} \int_{0}^{\frac{\pi}{4}} \exp \left(-\frac{g_{Q A M} \gamma}{\sin ^{2}(x)}\right) d x\right. \\
+ & \left.\int_{\frac{\pi}{4}}^{\frac{\pi}{2}} \exp \left(-\frac{g_{Q A M} \gamma}{\sin ^{2}(x)}\right) d x\right],
\end{aligned}
$$

where $g_{Q A M}=\frac{3}{2(M-1)}$. Furthermore, the average SER is derived from (24) as in [20], which is used for further analysis.

$$
\begin{aligned}
S E R_{a v g} & =2\left(1-\frac{1}{\sqrt{M}}\right)\left(1-\sqrt{\frac{g_{Q A M} \bar{\gamma}}{1+g_{Q A M} \bar{\gamma}}}\right) \\
& +\left(1-\frac{1}{\sqrt{M}}\right)^{2}\left[\frac{4}{\pi} \sqrt{\frac{g_{Q A M} \bar{\gamma}}{1+g_{Q A M} \bar{\gamma}}} \tan ^{-1} \sqrt{\frac{1+g_{Q A M} \bar{\gamma}}{g_{Q A M} \bar{\gamma}}}-1\right] .
\end{aligned}
$$

By following similar steps as we did to compute capacity in (23), the SER can be computed by substituting (2) and (22) in (27), and therefore, the behavior of channel in system's error performance can be found. The error performance analysis is reported in the next section.

\section{Simulation results}

Monte Carlo simulations are presented to evaluate BER and Cumulative Distribution Functions (CDFs) of SNR for the proposed IA approach. The BER performance for the long distance LoS MIMO $\mathrm{X}$ channel is analyzed for IA and non IA based transmission. Transmitted symbols $x_{11}, x_{21}, x_{12}$, and $x_{22}$ are drawn from a Quadrature Phase-Shift Keying (QPSK) modulation. Extensions to other modulations is straightforward. We assume that the power transmitted from each antenna is assumed to be equal to $P_{\text {ave }}$ and that $\sigma_{\text {LoS }}^{2}+$ $\sigma_{N L O S}^{2}=1$, so that the SNR at the receiver, defined as the average power of the received signal and the power of the Additive White Gaussian Noise (AWGN), is SNR $=\frac{N_{R} P_{\text {ave }}}{N_{T} \sigma_{n}^{2}}$.

First we consider the impact of IA based communication for a scheme with two HAPs and two GSs in overlapped coverage area. 




Fig. 2. BER versus SNR for IA and non IA scheme for $d_{R}$ less than $56.25 \mathrm{~m}$.

Eq. (18) is used for modeling MIMO channel in HAP-to-GS link, with AoA and AoD set within $10-30^{\circ}$ for all nodes due to high value of $R$ and $d_{T}=1 \mathrm{~m}$. As far as HAP is concerned, it would be reasonable to use node spacing, $d_{T}=1 \mathrm{~m}$. Figure 2 reports the BER performance of the IA and non IA based system for different receiver node separation distances. In order to achieve a fair comparison of IA system, where multiple transmitters and receivers form a communication system, a non IA system is realized, in which single transmitter sends data to a receiver via eigenmode transmission. Next, for analysis, we set the node separation at receiver and transmitter as $d_{R}=10,25$, and $55 \mathrm{~m}$ and $d_{T}=1 \mathrm{~m}$, respectively. For both IA and non IA, it can be seen that as $d_{R}$ is varied from $10 \mathrm{~m}$ to $25 \mathrm{~m}$, the performance improves and this trend continues even at $d_{R}=55$ $\mathrm{m}$. Hence, our result is in line with [10,21], that node separation at the receiver or transmitter provides independent channel further exploiting spatial multiplexing and diversity. It is clear that there is a drastic degradation in BER performance in IA in comparison with non IA case. Though there is a degradation in performance, this comes with an advantage in sum-rate, which can be seen in 4. However, advantage of IA persists due to higher degrees of freedom offered by IA systems. It turns out from the figure that, an inter node separation of $55 \mathrm{~m}$ shows better error performance than $d_{R}=10$ and $25 \mathrm{~m}$. This means that the nodes must be $56.2 \mathrm{~m}$ apart (from Eq. (7)) to achieve the best error performance. However, IA has poor performance with respect to non IA schemes, as IA requires orthogonal and independent channels in order to transmit independent symbols. In other words, though IA increases the capacity, the improvement comes at the price of error performance.

The node spacing $d_{R}$ is increased above the minimum node spacing found from (7) and BER performance is plotted in Fig. 3. When $d_{R}$ is set to 55,85 and $110 \mathrm{~m}$, a significant reduction in BER performance can be observed as $d_{R}$ increases for both IA and non IA systems. This is also in agreement with [12], which states the performance decreases above $\frac{\lambda R}{N d_{T}}$. Further investigation is done to observe the behavior at higher SNR values. From our simulations, it is possible to observe that a better BER performance is obtained when $n$ is an odd number, i.e. for $n=1$, and $d_{R}=56.25$ $\mathrm{m}$. This is because at $n=1,3, \ldots$, the correlation between columns of MIMO channel matrix is zero [11,12]. Similarly, both IA and non IA systems shows worst performance when $n$ is even, resulting in poorly correlated channel. This effect is due to the correlation factor between successive channels as explained in Section 2.3. By analyzing Figs. 2 and 3, it can be concluded that spacing nodes as per (7) provides the best BER performance, thus giving the optimal node separation at the receiver. The reason

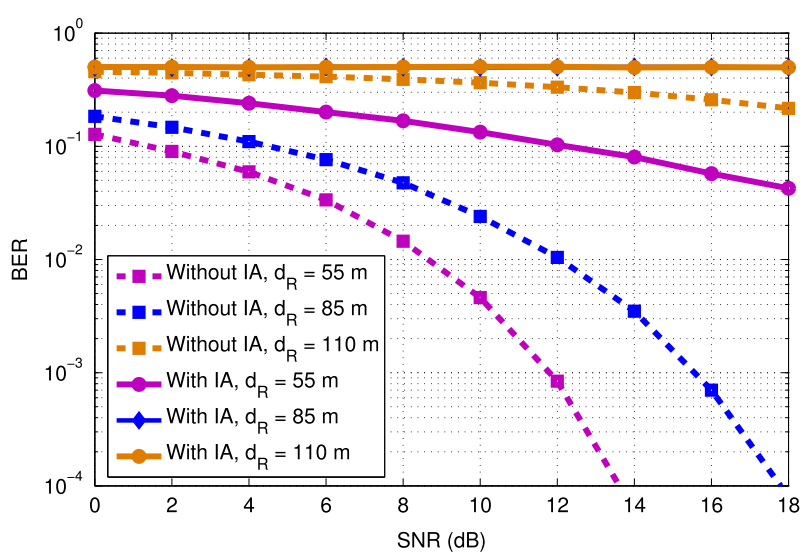

Fig. 3. BER versus SNR for IA and non IA scheme for $d_{R}$ greater than $56.25 \mathrm{~m}$



Fig. 4. CDF versus Rate for IA and non IA system with imperfect CSI.

behind such a heavy degradation in BER performance of IA system is that the independent channels are available only at $d_{R}^{o p t}$, where beamforming vectors in IA remain independent. It is worth noting that the optimal node spacing $d_{R}^{\text {opt }}$ remains valid for both IA and non IA based transmission, and this validates our proposed channel model with the one in [10]. The proposed scheme offers IA based transmission at any $d_{R}$, which makes the scheme more generic. However, the maximum capacity is obtained when $d_{R}=d_{R}^{o p t}$, which is derived using (7). In other words, to obtain maximum capacity in multiple HAP-to-GS communications, the HAPs must be separated by at least $d_{R}^{\text {opt }},(2 n+1) * 56.2 \mathrm{~m}$, where $\mathrm{n}$ is an integer.

In Fig. 4, we investigate the CDF of the sum-rate for the imperfect channel. CDF of the system is also analyzed using Monte Carlo simulations. Sum-rate for multiple iterations on the system defined by the Eqs. (12), (13), (16) and (17) are realized. In general, the figure shows the gain in sum-rate by using IA in the HAP-to-GS system. It can be seen that the average sum-rate has increased drastically in IA in comparison with non IA systems. Channel imperfection is represented by $\rho$. An SNR of $15 \mathrm{~dB}$ and $\kappa=-10 \mathrm{~dB}$ is considered to perform the analysis. The value $\rho=1$ implies perfect CSI while a lower value represents larger channel imperfection. From the figure, it is clear that sum-rate degrades with $\rho$. This is due to the fact that, IA performs perfectly with precoding and ZF matrices, designed from perfect CSI. Beamforming vectors designed from imperfect CSI deviate from the desired vectors and form non correlated vectors. Results show that a variation in locally available CSI, which is used to design ZF and precoding matrices, leads to a drastic reduction in the sumrate. Furthermore, the absence of precise station keeping will also lead to similar performance degradation. 


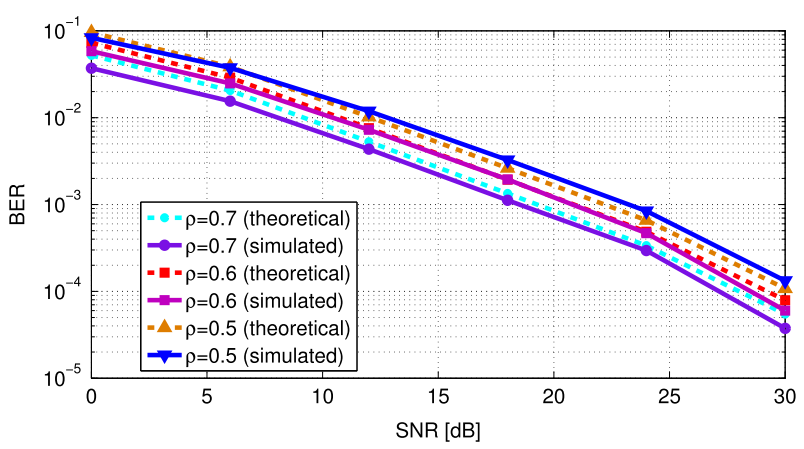

Fig. 5. BER versus SNR for different correlation coefficients.

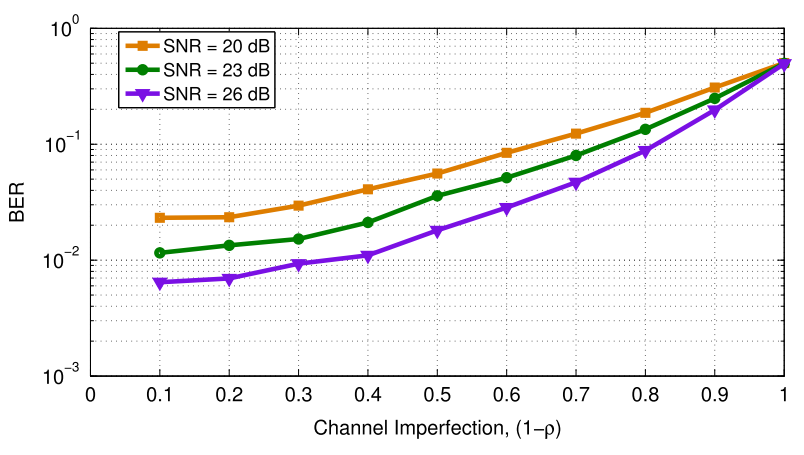

Fig. 6. BER versus channel imperfection $(1-\rho)$ for different SNRs.

Fig. 5 shows the effect of the imperfect channel on system performance. We consider $\kappa=-10 \mathrm{~dB}$ for this analysis. As $\rho$ increases, higher channel imperfection degrades error rate performance drastically. However, variation in error performance with higher $\rho$ is lesser, as compared to performance curves with smaller $\rho$. Analytical results for error analysis is also reported in Fig. 5. Equations (22) and (27) are used for plotting analytical BER. Analytical results matches with the simulated error performance.

In Fig. 6 we show the dependency of error performance on channel imperfection $(1-\rho)$ for different SNRs. $\rho=1$ corresponds to perfect CSI in the receiver. We consider $\kappa=-10 \mathrm{~dB}$ for this analysis, forming an LoS channel. From Fig. 6 it can be seen that error rate increases with channel imperfection. Furthermore, higher SNR value at receiver offers better error performance than lower ones. In other words, to overcome the effect of channel imperfection, the system must have higher transmission power. It is worth noting that the SNR used for the analysis is high. This is because the error performance of IA is worst in comparison with non IA schemes and analysis at high SNR is therefore required to understand the behavior of the system.

\section{Conclusion}

Interference Alignment achieves upper bound in DoF of an $X$ channel. Though IA in an $X$ channel provides higher sum-rate, performing IA in rank $1 \mathrm{MIMO}$ channels is a limiting factor. In this scenario, a new generalized MIMO channel model the consider AoA and AoD with an adequate transmitter-receiver separation has been proposed. Therefore, by carefully designing the system, it is possible to uncorrelated channels in the HAP-to-GS communication. This effectively overcomes the limitation to perform IA that is due to the rank 1 MIMO channel.
Furthermore, the optimal receiver separation for HAP communication in an $\mathrm{X}$ channel has been found. The optimal separation turns out to be the same for IA and non IA based communication. With IA being the key player in providing a higher sum-rate in an $\mathrm{X}$ channel, we propose IA based HAP-to-GS communication. The advantage of the proposed channel model is that IA can be successfully carried out due to the presence of spatially separated nodes and antennas. Considering the practical HAP deployment, the effect of imperfect CSI, which results from absence of precise HAP's stabilization, for multiple HAP-to-GS links is also investigated. An analytical expression is derived to analyze the error performance of the system. It is found that the system must operate at high SNR in order to compensate the effect of channel imperfection. Higher channel imperfection leads to a loss in the data rate.

\section{References}

[1] S.A. Jafar, et al., Interference alignment a new look at signal dimensions in a communication network, Found. Trends ${ }^{\circledR}$ Commun. Inf. Theory 7 (1) (2011) $1-134$.

[2] R. Prasad, S. Bhashyam, A. Chockalingam, On the sum-rate of the Gaussian MIMO Z channel and the Gaussian MIMO X channel, IEEE Trans. Commun. 63 (2) (2015) 487-497.

[3] M.A. Maddah-Ali, A.S. Motahari, A.K. Khandani, Communication over MIMO X channels: Interference alignment, decomposition, and performance analysis, IEEE Trans. Inform. Theory 54 (8) (2008) 3457-3470.

[4] S.A. Jafar, S. Shamai, Degrees of freedom region of the MIMO $x$ channel, IEEE Trans. Inform. Theory 54 (1) (2008) 151-170.

[5] V.R. Cadambe, S.A. Jafar, Interference alignment and the degrees of freedom of wireless $x$ networks, IEEE Trans. Inform. Theory 55 (9) (2009) 3893-3908.

[6] Y. Liu, D. Grace, P.D. Mitchell, Exploiting platform diversity for GoS improvement for users with different high altitude platform availability, IEEE Trans. Wireless Commun. 8 (1) (2009) 196-203.

[7] D. Grace, J. Thornton, G. Chen, G.P. White, T.C. Tozer, Improving the system capacity of broadband services using multiple high-altitude platforms, IEEE Trans. Wireless Commun. 4 (2) (2005) 700-709.

[8] A. Mohammed, A. Mehmood, F.-N. Pavlidou, M. Mohorcic, The role of highaltitude platforms (HAPs) in the global wireless connectivity, Proc. IEEE 99 (11) (2011) 1939-1953.

[9] D. Grace, M. Mohorcic, Broadband Communications via High-Altitude Platforms, John Wiley \& Sons, 2011.

[10] E. Torkildson, U. Madhow, M. Rodwell, Indoor millimeter wave MIMO: Feasibility and performance, IEEE Trans. Wireless Commun. 10 (12) (2011) 4150-4160.

[11] A. Irish, F. Quitin, U. Madhow, M. Rodwell, Sidestepping the rayleigh limit for los spatial multiplexing: A distributed architecture for long-range wireless fiber, in: Information Theory and Applications Workshop (ITA), 2013, IEEE, 2013, pp. 1-6.

[12] A. Irish, F. Quitin, U. Madhow, M. Rodwell, Achieving multiple degrees of freedom in long-range $\mathrm{mm}$-wave MIMO channels using randomly distributed relays, in: 2013 Asilomar Conference on Signals, Systems and Computers, IEEE, 2013, pp. 722-727.

[13] V.R. Cadambe, S.A. Jafar, Interference alignment and degrees of freedom of the $K$-user interference channel, IEEE Trans. Inform. Theory 54 (8) (2008) 3425-3441.

[14] P.G. Sudheesh, M. Magarini, P. Muthuchidambaranathan, Interference alignment in multiple-high altitude platforms based communication with a generalized long distance line-of-sight channel model, in: International Conference on Communication, Management and Information Technology, IEEE, 2017.

[15] A. Zajić, Mobile-to-Mobile Wireless Channels, Artech House, 2012.

[16] Y.S. Cho, J. Kim, W.Y. Yang, C.G. Kang, MIMO-OFDM Wireless Communications with MATLAB, John Wiley \& Sons, 2010.

[17] Y. Hu, Y. Hong, J. Evans, Modelling interference in high altitude platforms with 3D LoS massive MIMO, in: International Conference on Communications, (ICC), IEEE, 2016, pp. 1-6.

[18] R.F. Ustok, P.A. Dmochowski, P.J. Smith, M. Shafi, Aligned interference neutralisation for $2 \times 2 \times 2$ interference channel with imperfect channel state information, in: 2013 IEEE International Conference on Communications, (ICC), IEEE, 2013, pp. 5230-5235.

[19] S. Karapantazis, F. Pavlidou, Broadband communications via high-altitude platforms: A survey, IEEE Commun. Surv. Tutor. 7 (1) (2005) 2-31.

[20] M.K. Simon, M.-S. Alouini, Digital Communication Over Fading Channels Vol. 95, John Wiley \& Sons, 2005.

[21] F. Bohagen, P. Orten, G. Oien, Modeling and analysis of a $40 \mathrm{GHz}$ MIMO system for fixed wireless access, in: Vehicular Technology Conference, 2005 VTC 2005-Spring. 2005 IEEE 61st, Vol. 3, IEEE, 2005, pp. 1691-1695 


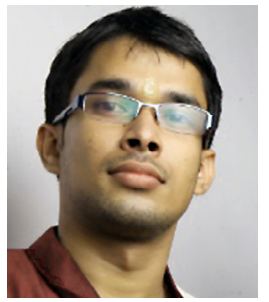

Sudheesh P.G. received his B.Tech Degree in Electronics and Communication Engineering from Mahatma Gandhi University, Kottayam, India, in 2010, the M.E. Degree in Communication Systems, from Anna University Chennai (Guindy campus), Chennai, India, in 2012. He is pursuing $\mathrm{Ph} . \mathrm{D}$. degree in from the National Institute of Technology (NIT), Tiruchirappalli, India from 2014. He worked as Assistant Professor at RSET, Cochin, during 2012-2014. His research interests include MIMO communication, channel modeling.

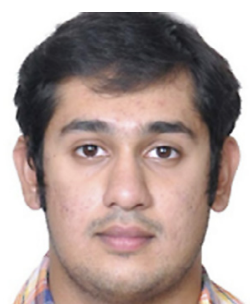

Navuday Sharma received Masters and Bachelors in Avionics (Dual Degree Program) from AMITY university, Noida. He is currently a PhD student in Politecnico di Milano, Italy from Oct 2015 . He also did research internship in Politecnico di Milano, Italy during Feb 2015-May 2015 and Indian Institute of Technology Bombay and Hindustan Aeronautical Limited in 2013 and 2012. His research interests include Information transmission, Wireless channel modeling and Unmanned aerial vehicles.

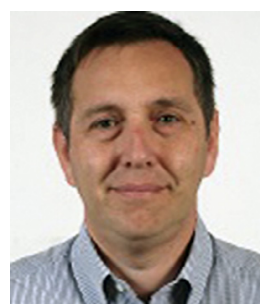

Maurizio Magarini was born in Milano, Italy, in 1969. He received the Master and Ph.D. degrees in electronic engineering from the Politecnico di Milano, Milano, Italy, in 1994 and 1999, respectively. In 1994, he was granted the TELECOM Italia scholarship award for his Master thesis. From 1999 to 2001 he was a Research Associate in the Dipartimento di Elettronica e Informazione at the Politecnico di Milano where, since 2001, he has been an Assistant Professor. From August 2008 to January 2009 he spent a sabbatical leave at Bell Labs, Alcatel-Lucent, Holmdel, NJ. He has authored and coauthored more than 40 journal and conference papers. His research interests are in the broad area of communication theory. Topics include synchronization, channel estimation, equalization, coding and reduced complexity detection schemes for multi-antenna systems.

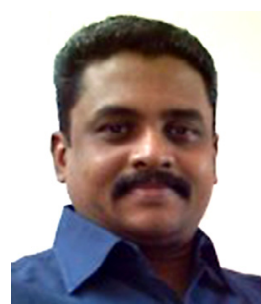

P. Muthuchidambaranathan received his B.Eng. Degree in Electronics and Communication Engineering from Government College of Technology, Coimbatore, India, in 1992, the M.Eng. Degree in Microwave and Optical Engineering, from A.C. College of Engineering and Technology, Karaikudi, India, in 1994. He obtained his Ph.D. degree in optical communication from the National Institute of Technology (NIT), Tiruchirappalli, India in 2009. He is currently working as an Associate Professor in the Department of Electronics and Communication Engineering, National Institute of Technology (NIT), Tiruchirappalli, India. His research interests include wireless communications, and optical communications. He published his research papers in refereed international journals, and international and national conferences. He is an author of the textbook "Wireless Communications" (published by Prentice Hall of India). 